\title{
An Example of a Hybrid System: Coherent Optical System with Costas Loop in Receiver-System for Transmission in Baseband
}

\author{
I. B. Djordjević, M. C. Stefanović, Associate Member, IEEE, S. S. Ilić, and G. T. Djordjević
}

\begin{abstract}
The possibility of hybrid system construction is considered in this paper. The coherent optical system is the constituent part of this system. The first part of the system is constructed in passband (optical cable is transmission medium), and the second part of the system is constructed in baseband. The heterodyne optical receiver is used as optical receiver. Estimation is not done just after demodulation, but it is done at the end of the section in baseband. Taking into account shot noise of photodetectors, laser phase noise (transmitter laser and local laser) and noise which is generated during transmission by means of the section in baseband, we determine the performances of the proposed system.
\end{abstract}

Index Terms - Coherent optical systems, Costas loop, hybrid system.

\section{INTRODUCTION}

$\mathbf{T}$ HE construction of the hybrid system which contains the coherent optical system is considered in this paper. The proposed system consists of two sections. Signal is transmitted in passband by means of the first section (optical cable), and in baseband by means of the second section (coaxial cable). Digital amplitude modulation is applied. The heterodyne receiver with Costas loop for carrier refresh is used (as receiver). Costas loop is used due to its greater tolerance to laser phase noise in comparison with decisiondriven loop (DDL) [1]. Sampling is made at the end of the section in passband, but decision estimation is not done there. Amplitude shift keying (ASK) receiver output sampling signal is transmitted by coaxial cable. Filtering, repeated sampling and estimation are done at the end of the section in baseband.

The shot noise of photodetectors, laser phase noise (transmitter laser and local laser) and noise which is generated during transmission by means of the section in baseband are taken into consideration. The laser phase noise is considered in the specific way [2].

This paper differs from the other ones (for example, [3]-[5]), because the laser phase noise is presented as the frequency shift of transmitter laser carrier frequency, and the frequency shift of local laser basic frequency. The shot noise is considered in the classic way as in [1]-[5]. In addition, the laser phase noise and other kinds of noise and disturbances (photodiode

Manuscript received November 7, 1996; revised July 24, 1997.

The authors are with the Faculty of Electronic Engineering, University of Nis, Nis 18000 Yugoslavia.

Publisher Item Identifier S 0733-8724(98)01235-3. shot noise) are described through loop parameter variations [the parameters $\alpha$ and $\beta$ in (19), (22), and (25)].

\section{HETERODYNE OPTICAL RECEIVER WITH COSTAS LoOP}

The system model is shown in Fig. 1(a) and (b).

The received optical signal

$$
S=\sqrt{2 P_{S}} d(t) \cos \left[\omega_{S} t+\psi_{S}(t)\right]
$$

is treated by the receiver. The received optical signal power and frequency are denoted by $P_{S}$ and $\omega_{S}$, respectively, $\psi_{S}$ is the transmitter laser phase noise, and $d(t) \in\{0,1\}$ is the information contents. The receiver contains the local oscillatorlaser whose output is

$$
L=\sqrt{2 P_{\mathrm{LO}}} \cos \left[\omega_{\mathrm{LO}} t+\psi_{\mathrm{LO}}(t)\right]
$$

where $P_{\mathrm{LO}}, \omega_{\mathrm{LO}}$, and $\psi_{\mathrm{LO}}$ are the local laser power, frequency and phase noise, respectively. The optical signals are transmitted to the $3 \mathrm{~dB}$-coupler whose outputs signals excite two photodiodes. If the balanced receiver is ideal, the output current $i_{T}(t)$ is

$$
i_{T}(t)=2 R \sqrt{P_{S} P_{\mathrm{LO}}} d(t) \cos \left[\omega_{\mathrm{IF}} t+\psi(t)\right]+n(t)
$$

where $R$ is the photodiode responsitivity, $\omega_{\mathrm{IF}}$ is the difference between received optical signal frequency and local laser output frequency, $\omega_{\mathrm{IF}}=\left|\omega_{S}-\omega_{\mathrm{LO}}\right|, d(t) \in\{0,1\}$, and $\psi(t)$ is the total laser phase noise. $n(t)$ is the shot noise with double power spectral density

$$
S_{n}(f)=R q\left(P_{S}+P_{\mathrm{LO}}\right)
$$

where $q$ is an electron charge. $P_{\mathrm{LO}} / P_{S}$ is usually greater than 0.001 , so PSD of the shot noise $n(t)$ can be approximated by $q R P_{\mathrm{LO}}$. The IF amplifier output signal, under the assumption that filter is ideal with bandwidth $2 B=2 R_{b}$ ( $R_{b}$ is the system bit rate), (this corresponds to the PSD main lobe), is

$$
V(t)=2 G_{A} R \sqrt{P_{S} P_{\mathrm{LO}}} d(t) \cos \left[\omega_{\mathrm{IF}} t+\psi(t)\right]+n_{\mathrm{IF}}(t)
$$

where $G_{A}$ is the IF amplifier amplification, and $n_{\mathrm{IF}}(t)$ is the passband noise.

The constituent part of the receiver is also the electronic IF VCO (voltage controlled oscillator), whose output voltage is

$$
V_{\mathrm{VCO}}(t)=A_{\mathrm{VCO}} \cos \left[\omega_{\mathrm{IF}} t+\phi_{\mathrm{VCO}}(t)\right]
$$




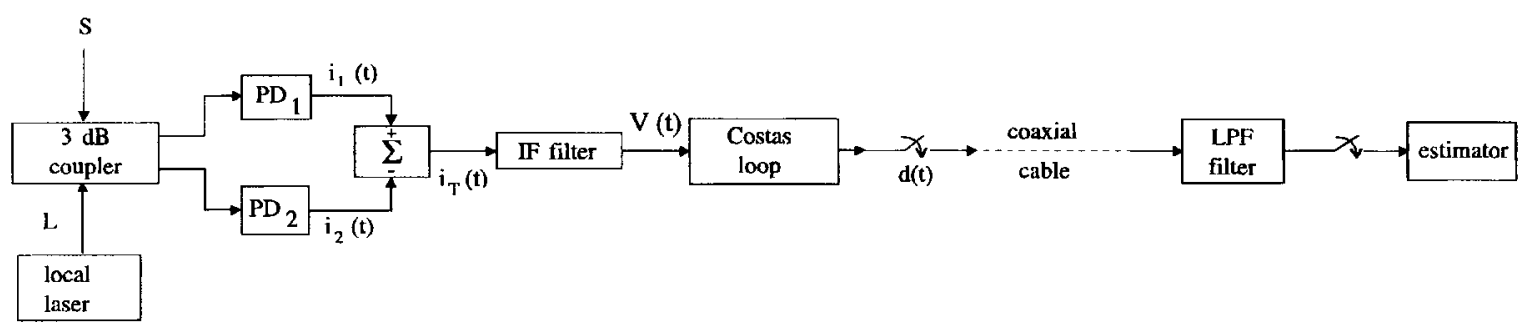

(a)

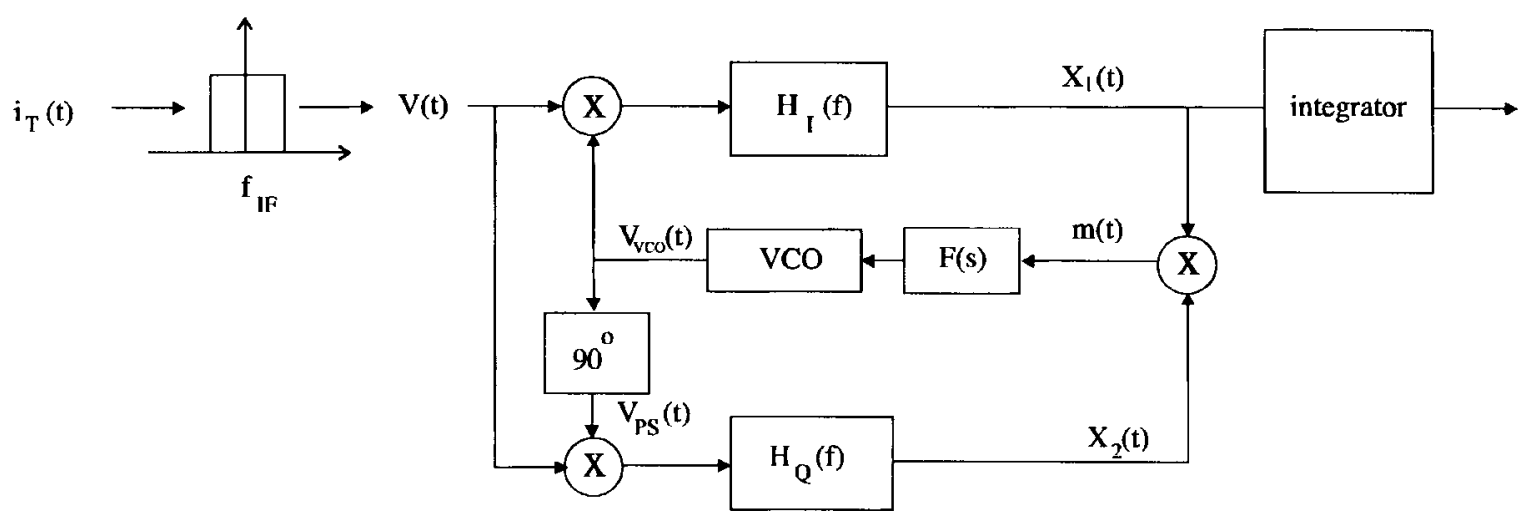

(b)

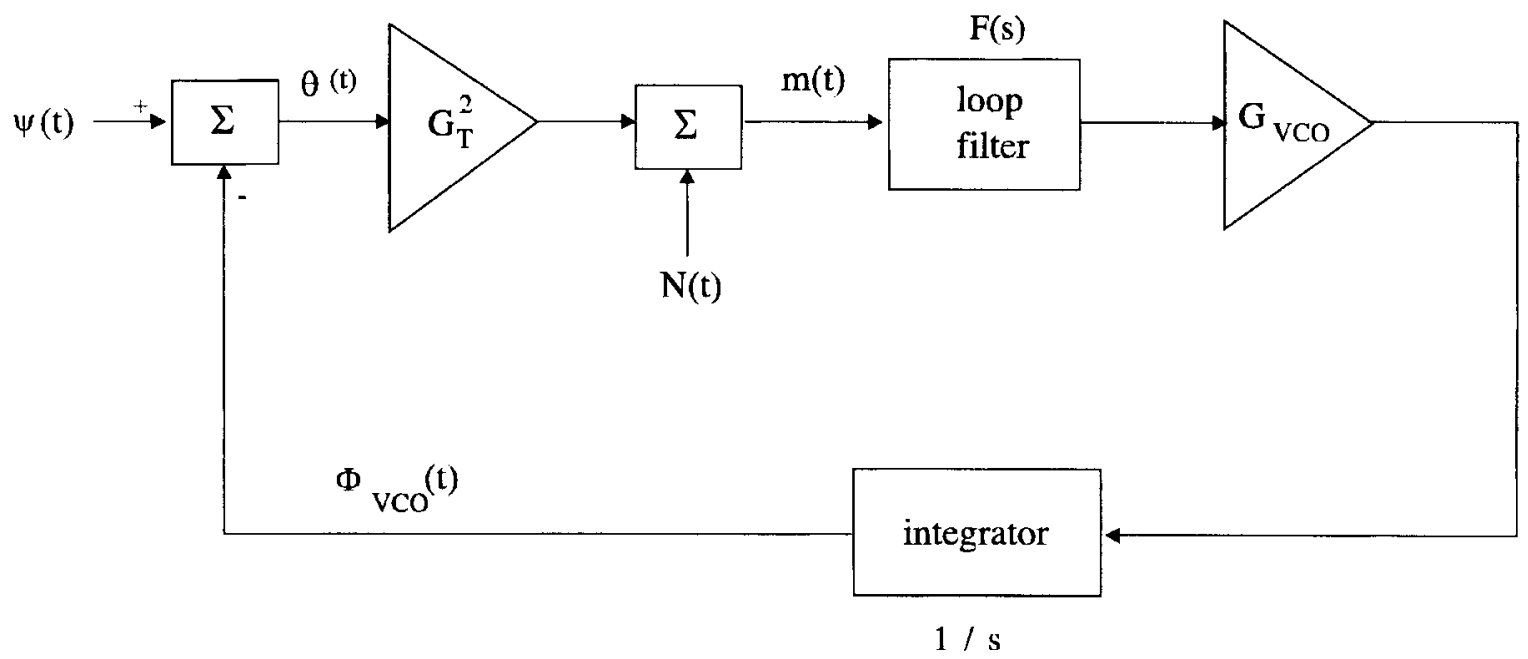

(c)

Fig. 1. Block scheme of hybrid system: (a) receiver model, (b) Costas loop model, and (c) Costas loop linear model.

where $A_{\mathrm{VCO}}$ and $\phi_{\mathrm{VCO}}$ are the amplitude and phase of the VCO output signal, respectively. Voltage controlled oscillator output signal phase is

$$
\phi_{\mathrm{VCO}}(t)=G_{\mathrm{VCO}} \int_{-\infty}^{t} V_{C}\left(t^{\prime}\right) d t^{\prime}
$$

where $G_{\mathrm{VCO}}(\mathrm{rad} / \mathrm{V} / \mathrm{s})$ is the VCO amplification. The phase shifter output is

$$
V_{P S}(t)=-A_{\mathrm{VCO}} \sin \left(\omega_{\mathrm{IF}} t+\phi_{\mathrm{VCO}}\right) .
$$

The low-pass filters outputs are

$$
X_{1}(t)=A_{\mathrm{VCO}} R d(t) G_{A} G_{M} \sqrt{P_{S} P_{\mathrm{LO}}} \cos \theta(t)+n_{p}(t)
$$
and

$$
X_{2}(t)=A_{\mathrm{VCO}} R d(t) G_{A} G_{M} \sqrt{P_{S} P_{\mathrm{LO}}} \sin \theta(t)+n_{q}(t)
$$

where $\theta(t)=\psi(t)-\phi_{\mathrm{VCO}}(t)$ is the total phase error, $G_{M}$ is the multiplier constant; $n_{p}(t)=n_{\mathrm{IF}}(t) V_{\mathrm{VCO}}(t)$ and $n_{q}(t)=$ $n_{\mathrm{IF}}(t) V_{\mathrm{VCO}}(t) e^{j \pi / 2}$ are the noise components in-phase and in-quadrature. $n_{\mathrm{FF}}(t)$ is the passband process. By assumption, the low-pass filters $H_{I}(f)$ and $H_{Q}(f)$ have the rectangular spectral characteristic with double passband width $2 B=2 R_{b}$. The multiplier output signal can be written as

$$
\begin{aligned}
m(t) & =X_{1}(t) X_{2}(t)=S(t)+N(t) \\
& =\frac{G_{T}^{2}}{2} \sin 2 \theta(t)+S_{p} n_{q}+n_{p} S_{q}+n_{p} n_{q}
\end{aligned}
$$

where $S_{q}=G_{T} d(t) \sin \theta(t)$, and $S_{p}=G_{T} d(t) \cos \theta(t)$. The phase error signal is denoted by $S(t), N(t)$ is the total noise; while $G_{T}=A_{\mathrm{VCO}} R G_{A} G_{M} \sqrt{P_{S} P_{\mathrm{LO}}}$. In purpose to simplify the analysis, we assume that the loop is in lock-in-range for 
little phase errors $(\theta(t) \ll 1)$. The sine of little argument can be approximated by the argument $(\sin x \cong x$, for $x \rightarrow 0$ ), so the previous expression becomes

$$
m(t) \cong G_{T}^{2} \theta(t)+N(t) .
$$

On the basis of previous expression, the linear loop model is shown in Fig. 1(c).

The total phase error variance can be determined as [1]

$$
\begin{aligned}
\sigma_{\theta}^{2} & =\sigma_{N}^{2}+\sigma_{\psi}^{2} \\
& =B_{n}\left(\frac{q}{R P_{S}}+\frac{q^{2} B}{R^{2} P_{S}^{2}}\right)+\frac{\pi \Delta \nu\left(1+4 \varsigma^{2}\right)}{8 \varsigma^{2} B_{n}}
\end{aligned}
$$

where the first term $\sigma_{N}^{2}$ corresponds to the noise contribution $N(t)$ [from (10)], and the second one $\sigma_{\psi}^{2}$ corresponds to the laser phase noise contribution to the total phase error variance. The loop damping factor is denoted by $\varsigma$ (this parameter regulates the loop calming down rate, i.e., stationary regime beginning rate), and $B_{n}$ is the loop noise bandpass width. From (12), it is evident that when $B_{n}$ increases, then $\sigma_{N}^{2}$ also increases, while $\sigma_{\psi}^{2}$ decreases; so there is the optimal $B_{n}$ value for which the total phase error variance $\sigma_{\theta}^{2}$ is minimum. This optimal value is

$$
B_{n, \mathrm{opt}}=\sqrt{\frac{3 \pi \Delta \nu / 4}{\frac{q}{R P_{S}}+\frac{q^{2} B}{R^{2} P_{S}^{2}}}}
$$

for $\zeta=0.707$ (this damping factor value is almost used in literature [1] and is caused by the compromise between phase loop stationary state beginning rate and minimum phase error variance). By substituting this $B_{n \text { opt }}$ in (12) for $\zeta=0.707$, the minimum phase error variance value is

$$
\sigma_{\min }^{2}=\sqrt{3 \pi \frac{\Delta \nu}{R_{b}} \frac{1}{p m}\left(1+\frac{1}{p m}\right)}
$$

where $m=R P_{S} /\left(q R_{b}\right)=\eta P_{S} /\left(h \nu R_{b}\right)$ is the number of photons per a bit, and $\eta$ is the quantum efficiency (by assumption $\eta=1$ ).

The phase error standard deviation $\sigma$ (in degrees) dependence on normalized loop bandwidth $B_{n} / \Delta \nu$ for several values of power-to-linewidth ratio (PLR $=P_{n} / \Delta \nu$, where $\left.P_{n}=R P_{S} / q\right)$ is shown in Fig. 2.

It is evident that condition $\sigma_{\min }<10^{\circ}$ can be achieved whenever PLR is greater than $10^{4}$. It is easy to show that when the Costas loop is applied the following requirement is valid: $\Delta \nu \leq 2.29 \cdot 10^{-3} R_{b}$ for $\mathrm{BER}=10^{-10}$ and total power penalty $=0.5 \mathrm{~dB}$, and in case of DDL applications is $\Delta \nu \leq 2.26 \cdot 10^{-3} R_{b}$ for the same BER and power penalty as before [1]. It can be noticed that the Costas loop is little more tolerant than the DDL in heterodyne PSK system, considering the phase noise. Because of this, we use the coherent optical receiver with Costas loop in this paper.

$X_{1}(t)$ is filtered by LPF filter, which can be presented as integrator with the integration constant equal to the bit duration. The sampling circuit output signal (before than signal

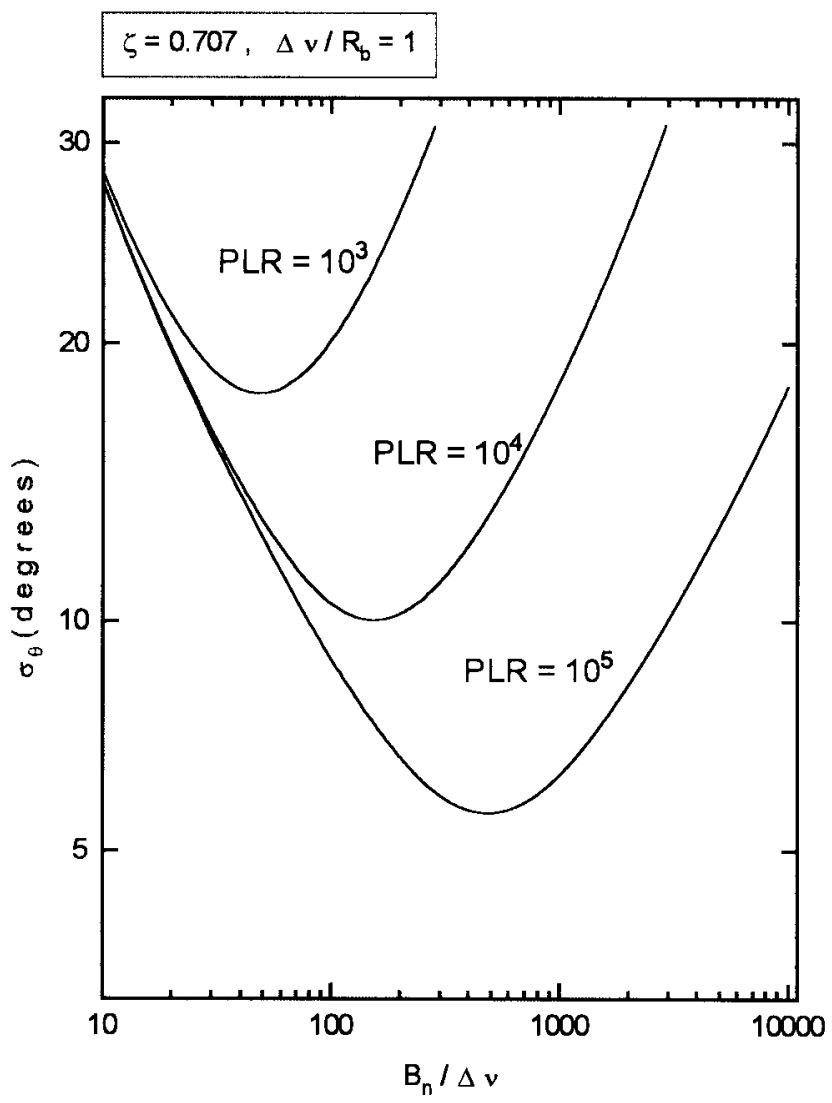

Fig. 2. Phase error standard deviation $\sigma_{\theta}$ (in degrees) versus the normalized loop band width $B_{n} / \Delta \nu$ for several values of the power-to-linewidth ratio PLR.

is transmitted by means of coaxial cable) is written as

$$
\begin{aligned}
z(k T)= & \frac{A_{\mathrm{VCO}} R d(k T) G_{A} G_{M} \sqrt{P_{S} P_{\mathrm{LO}}}}{T} \int_{0}^{T} \cos \theta(t) d t \\
& +\frac{1}{T} \int_{0}^{T} n_{p}(t) d t .
\end{aligned}
$$

The second term in the expression (15) is suitable for analysis because it is a zero-mean Gaussian process with variance

$$
\sigma_{n}^{2}=\int_{-B_{n_{\mathrm{opt}}}}^{B_{n_{\mathrm{opt}}}} S_{n_{p}}(f)\left|H_{i}(f)\right|^{2} d f
$$

where $H_{i}(f)$ is the integrator transmission function $\left|H_{i}(f)\right|^{2}=\left(\sin ^{2} \pi f T / \pi^{2} f^{2} T^{2}\right) .2 B_{n_{\text {opt }}}$ is the filter bandwidth where $B_{n_{\text {opt }}}$ corresponds to the optimal loop bandwidth. Similarly, as in [1], $S_{n_{p}}(f)=$ $0.5 A_{\mathrm{VCO}}^{2} q R G_{A} P_{\mathrm{LO}}$, it is

$$
\sigma_{n}^{2}=A_{\mathrm{VCO}}^{2} q R G_{A} P_{\mathrm{LO}} \int_{0}^{B_{n_{\mathrm{opt}}}}\left|H_{i}(f)\right|^{2} d f .
$$

For moderate values of loop noise bandwidth $B_{n}$ $\left(B_{n}<0.15 R_{b}\right), \theta(t)$ is slowly changeable, so $z(k T)$ is approximated in the following way:

$$
z(k T)=A_{\mathrm{VCO}} R d(k T) G_{A} G_{M} \sqrt{P_{S} P_{\mathrm{LO}}} \cos \theta+n(k T) .
$$




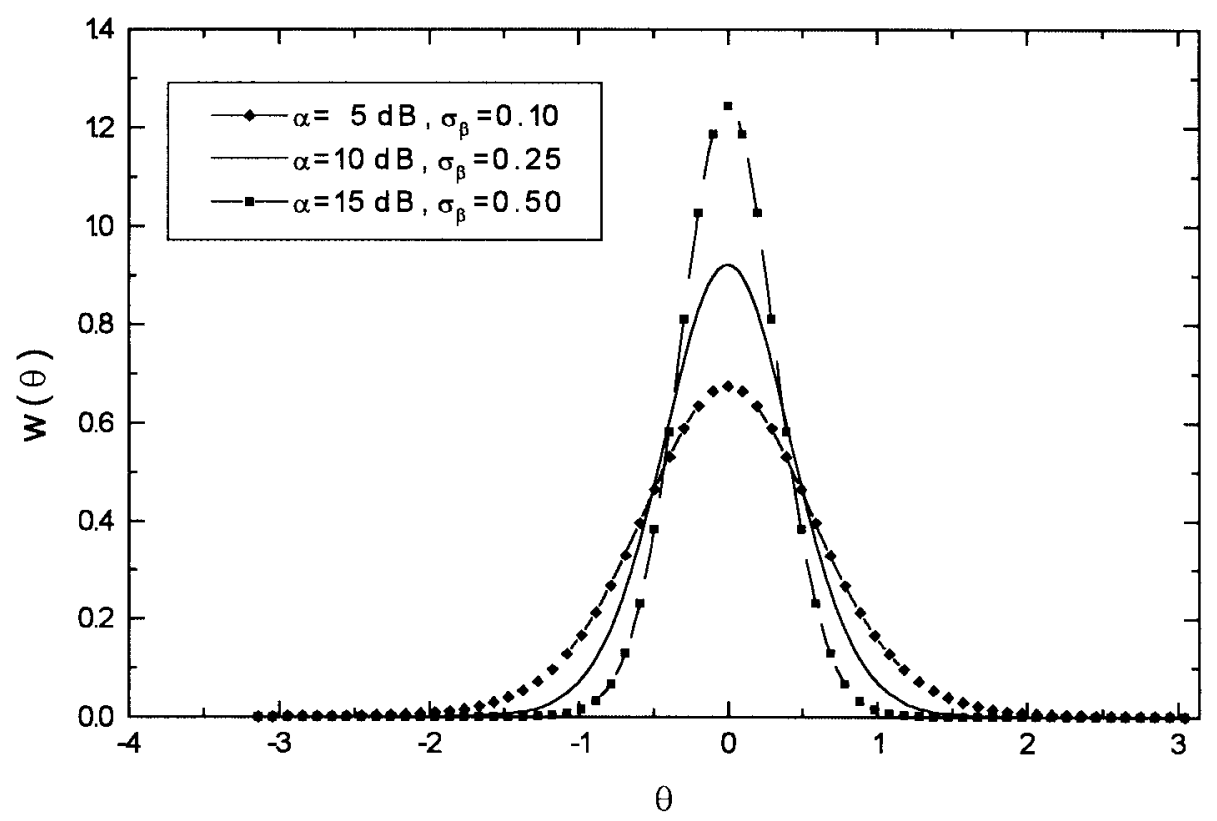

Fig. 3. The pdf for total phase error: $\alpha=10 \log \left(2 A / k N_{0}\right)$ [dB] and $\sigma_{\beta}$ are used as parameters.

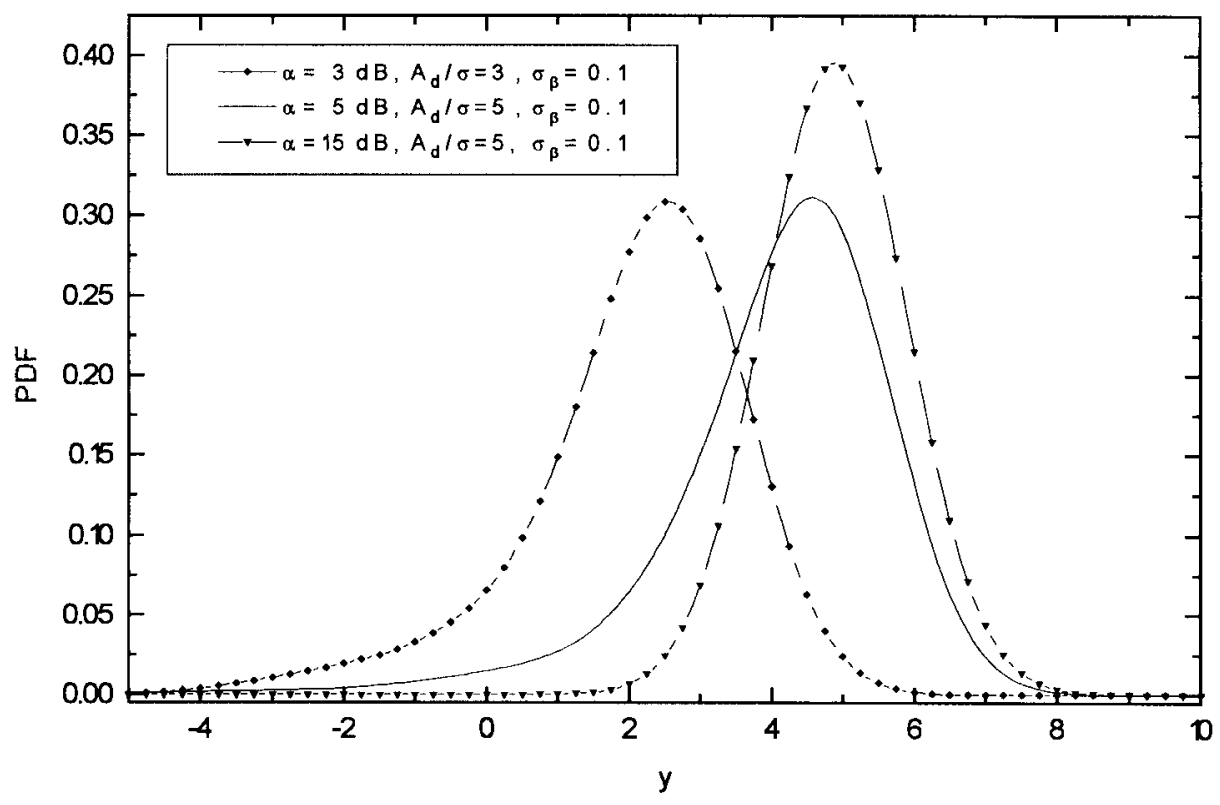

Fig. 4. Probability density function for stochastic variable $Y$ when $a_{k}=1$.

The sampler before the section in baseband makes possible the signal transmission through coaxial cable to be in digital form.

\section{Phase ERror Probability DENSITY FUNCTION DETERMINATION}

The conditional probability density function of the phase error in the presence of the phase noise can be written as [6]

$$
\begin{aligned}
w(\theta / \beta)= & C \exp (\alpha \cos \theta+\beta \theta) \\
& \cdot\left[1+D \int_{\pi}^{\theta} e^{-(\alpha \cos x+\beta x)} d x\right], \quad|\theta| \leq \pi
\end{aligned}
$$

where

$$
\begin{aligned}
& \alpha=2 A /\left(k N_{0}\right) \\
& D=e^{-2 \pi \beta}-1 / \int_{-\pi}^{\pi} e^{-(\alpha \cos x+\beta x)} d x \\
& \beta=2\left(\omega_{\mathrm{IF}}-\omega_{0}\right) /\left(k^{2} N_{0}\right) .
\end{aligned}
$$

In addition, pdf for $\dot{\phi}(\dot{\phi}$ denotes the influence of the laser phase noise) is [4]

$$
w(\dot{\phi})=\frac{1}{\sigma_{\psi} \sqrt{2 \pi}} e^{-\left(\dot{\phi}^{2} / 2 \sigma_{\psi}^{2}\right)} .
$$




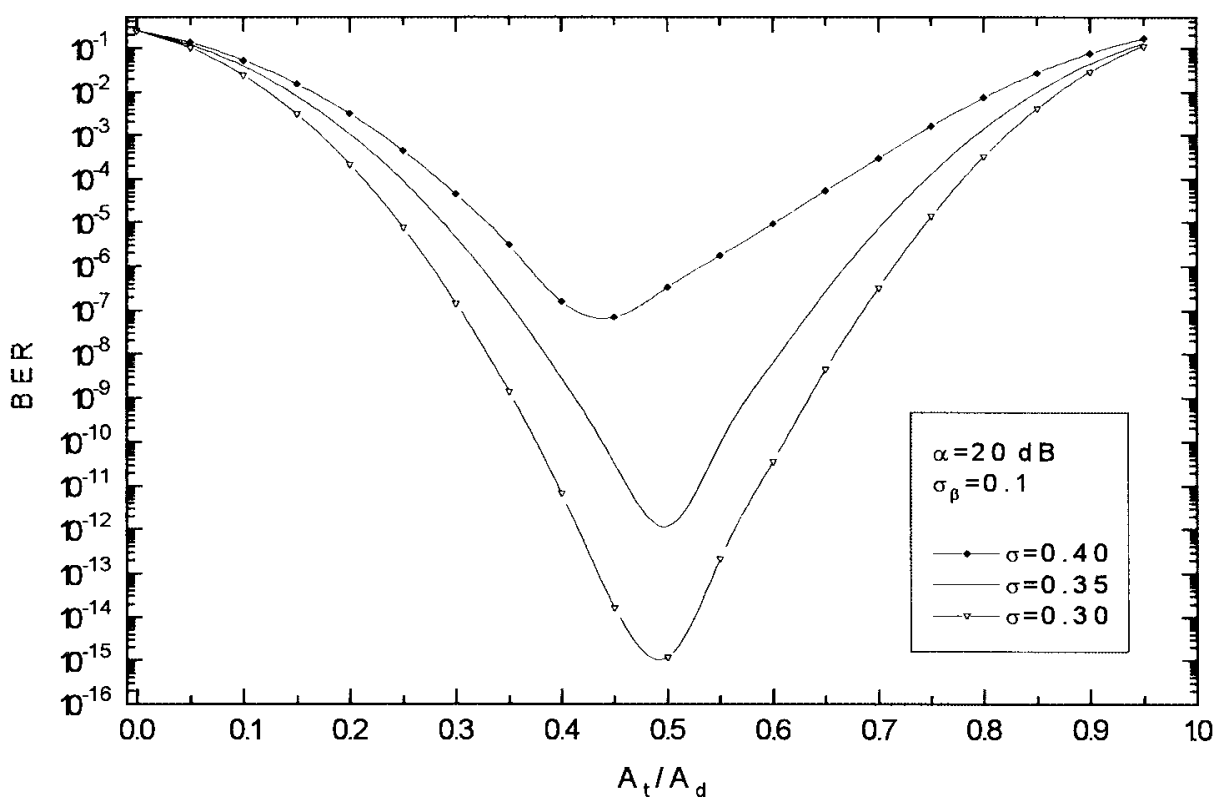

Fig. 5. Error probability dependence on normalized threshold.

When PLL is synchronized

$$
\omega_{\mathrm{IF}}=\omega_{0}+\dot{\phi}
$$

So

$$
\beta=2 \dot{\phi} /\left(k^{2} N_{0}\right)
$$

Then, pdf for $\beta$ becomes

$$
w(\beta)=\frac{1}{\sigma_{\beta} \sqrt{2 \pi}} e^{-\left(\beta^{2} / 2 \sigma_{\beta}^{2}\right)}
$$

where

$$
\sigma_{\beta}=\frac{2 \sigma_{\psi}}{k^{2} N_{0}}
$$

The probability density function of the phase error in the presence of the laser phase noise can be expressed now as

$$
\begin{aligned}
w(\theta)=C e^{\alpha \cos \theta} & {\left[\int_{-\infty}^{\infty} e^{\beta \theta} w(\beta) d \beta+\int_{-\infty}^{\infty} D e^{\beta \theta} w(\beta)\right.} \\
& \left.\cdot \int_{\pi}^{\theta} e^{-(\alpha \cos x+\beta x)} d x d \beta\right]
\end{aligned}
$$

where

$$
\begin{array}{r}
C=\left\{\int _ { - \pi } ^ { \pi } e ^ { \alpha \operatorname { c o s } \theta } \left[\int_{-\infty}^{\infty} e^{\beta \theta} w(\beta) d \beta+\int_{-\infty}^{\infty} D e^{\beta \theta} w(\beta)\right.\right. \\
\left.\left.\cdot \int_{\pi}^{\theta} e^{-(\alpha \cos x+\beta x)} d x d \beta\right] d \theta\right\}^{-1} .
\end{array}
$$

$A=2 G_{A} R \sqrt{P_{S} P_{\mathrm{LO}}}$ and $\omega_{\mathrm{IF}}$ are the amplitude and frequency of the Costas loop incoming signal, $\omega_{0}$ is the VCO own frequency, $k=A_{\mathrm{VCO}}$ is the VCO output signal amplitude, $\theta(t)$ is difference between the incoming signal phase and reconstructed signal phase, while $\dot{\phi}$ is the influence of the laser phase noise and $N_{0}=q R\left(P_{S}+P_{\mathrm{LO}}\right) G_{A}^{2}$ is the passband noise double power spectral density.

\section{System Performance Determination}

The useful signal at the beginning of the section in baseband is presented as $a_{k} A_{d} \cos \theta$ where $a_{k} \in\{0,1\}$, and $A_{d}=$ $A_{\mathrm{VCO}} R G_{A} G_{M} \sqrt{P_{S} P_{\mathrm{LO}}}$.

A zero mean Gaussian noise with variance $\sigma_{c}^{2}$ is added to useful signal at the end of the section in baseband. Therefore, the variance of the resulting Gaussian noise at the estimation point is $\sigma^{2}=\sigma_{n}^{2}+\sigma_{c}^{2}$. Hence, the probability density function for the stochastic variable

$$
Y=\left(\sum_{k=-\infty}^{\infty} z(k T) \delta(t-k T)+n_{c}(t)\right) * h_{\mathrm{LPF}}(t)
$$

at the end of the section in baseband is

$$
\operatorname{pdf}_{a_{k}}(y)=\frac{1}{\sigma \sqrt{2 \pi}} \int_{-\pi}^{\pi} e^{-\left(\left(y-a_{k} A_{d} \cos \theta\right)^{2} / 2 \sigma^{2}\right)} w(\theta) d \theta
$$

where $w(\theta)$ is the pdf for the total phase error (25) (Fig. 3). $h_{\mathrm{LPF}}(t)$ is the impulse response of the low-pass filter at the output of the coaxial cable, * denotes the convolution and $n_{c}(t)$ is the additive Gaussian white noise which corrupts the useful signal during the transmission through coaxial cable. This lowpass filter has the role only to limit the noise power spectral density inside the useful signal power spectral density range.

The pdf of $Y$ for different values of parameters $A_{d} / \sigma$, $\alpha=10 \log \left(\left(4 \sqrt{\left.\left(P_{S} P_{\mathrm{LO}}\right)\right)} /\left(A_{\mathrm{VCO}} q\left(P_{s}+P_{\mathrm{LO}}\right) G_{A}\right)[\mathrm{dB}]\right.\right.$ and for $\sigma_{\beta}=0.1$ is shown in Fig. 4 . 


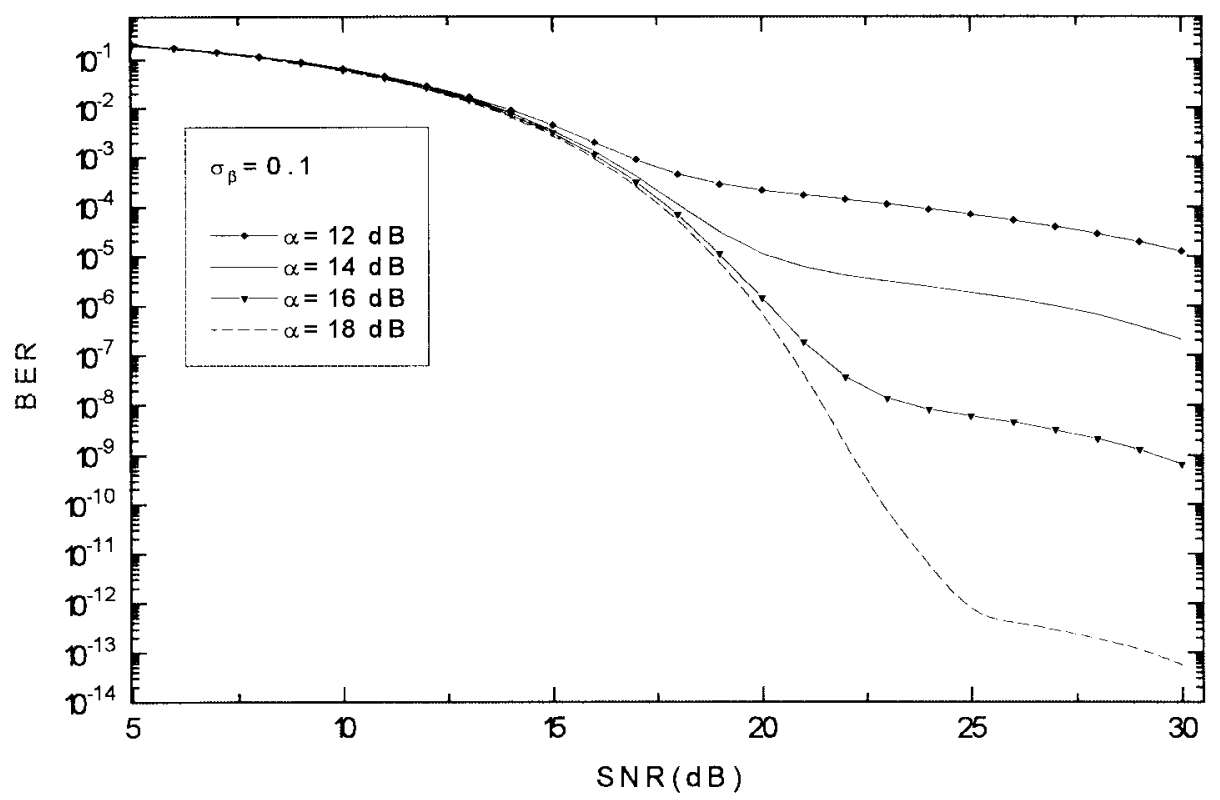

Fig. 6. Error probability for hybrid system. $\mathrm{SNR}=20 \log \left(A_{d} / \sigma\right)$.

If we determine the optimal threshold, we can compute the error probability. The optimal threshold is determined from error probability dependence on normalized threshold. It is found that the average value of the optimal threshold is $0.5 A_{d}$. This is illustrated in Fig. 5.

The error probability can be determined numerical by starting from

$$
\mathrm{BER}=\frac{1}{2}\left(\int_{-\infty}^{0.5 A_{d}} \operatorname{pdf}_{1}(y) d y+\int_{0.5 A_{d}}^{+\infty} \operatorname{pdf}_{0}(y) d y\right)
$$

The error probability dependence on signal to noise ratio for four values of parameters and $\alpha$ and $\sigma_{\beta}=0.1$ is shown in Fig. 6.

\section{CONCLUSION}

The hybrid system: heterodyne coherent optical system with Costas loop in receiver-system for transmission in baseband is investigated in this paper. On the basis of the loop linear model, the expression of the phase error variance is originally evaluated. The minimum phase error standard deviation $\left(\sigma_{\mathrm{opt}}\right)$ which is less than $10^{\circ}$ can be achieved whenever power-tolinewidth ratio (PLR) is greater than $10^{4}$. The coherent optical system (the first section) with the Costas loop as its constituent part is considered here, due to the Costas loop greater tolerance to the phase noise in comparison with the decision-driven loop.

The shot noise and laser phase noise (of transmitter and local laser) are taken into consideration. The laser phase noise is specifically presented, as instantaneous laser beam frequency variations of both transmitter laser and local laser. The laser phase noise and photodiode shot noise are shown as loop parameter variations ( $\alpha$ and $\beta$ ). The phase error pdf is obtained by averaging conditional pdf per variable loop parameters.
Average optimal threshold $\left(0.5 A_{d}\right)$ is determined by averaging threshold values which are obtained when the probability error is minimum, for different values of system parameters.

Based on the probability error values for observed hybrid system, there is the possibility of its application in local telecommunication networks, where the coaxial cable length is not too great, and error probability of optical system part is less than $10^{-10}$. BER floor appears as a consequence of the laser phase noise and can be reduced by increasing the transmitter laser power.

\section{REFERENCES}

[1] I. B. Djordjevic, "Performances of coherent optical systems with PLL," Master thesis, Faculty Electron. Eng., Univ. of Nis, Yugoslavia, pp. 102-108, 1996.

[2] M. C. Stefanovic, I. B. Djordjevic, and Z. H. Peric, "Performances of the ASK system with optical PLL in presence of laser phase noise," $J$. Optic. Commun., vol. 18, no. 3, pp. 114-116, June 1997.

[3] L. G. Kazovsky, "Performance analysis and laser linewidth requirements for optical PSK heterodyne communications systems," J. Lightwave Technol., vol. LT-4, pp. 415-425, Apr. 1986.

[4] J. Salz, "Coherent lightwave communications," AT\&T Tech. J., vol. 64 no. 10, pp. 2153-2209, Dec. 1985.

[5] S. Huang and L. Wang, "Exact evaluation of laser linewidth requirements for optical PSK homodyne communication system with balanced PLL receivers," J. Lightwave Technol., vol. 14, pp. 661-664, May 1996.

[6] N. R. Levin, Teoreticheskie Osnovy Satisticheskoj Radiotehniki, Sovetskoe radio, pp. 405-407, Moscow, Russia, 1974.

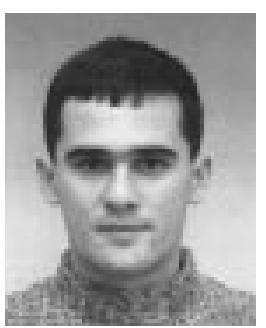

I. B. Djordjević was born in Nis, Yugoslavia, on December 11, 1969. He received the B.E. and M.E. degrees in electrical engineering from the Faculty of Electronic Engineering, University of Nis in 1994 and 1996, respectively.

His research interests include information theory, coding theory, and optical communication systems and their applications. 


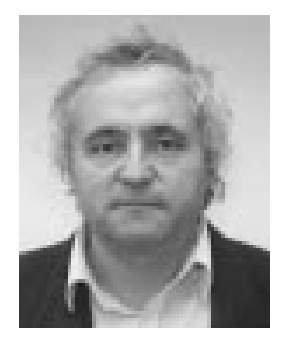

M. C. Stefanović (A'92) was born in Nis, Yugoslavia, in 1947. He received the B.E., M.E., and $\mathrm{Ph} . \mathrm{D}$. degrees in electrical engineering from the Faculty of Electronic Engineering, University of Nis, Nis, in 1971, 1976, and 1978, respectively.

His primary research interests are statistical communication theory, optical, and satellite communications.

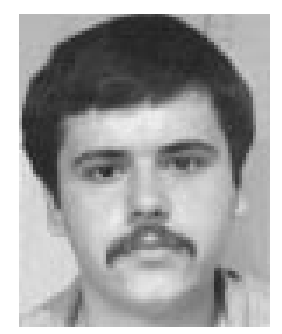

S. S. Ilić was born in Nis, Yugoslavia, on July 24 , 1970. He received the B.E. degree in electrical engineering from the Faculty of Electronic Engineering, University of Nis in 1995.

His areas of interest include applied electromagnetics and optical communications.

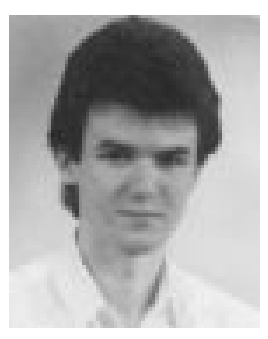

G. T. Djordjević was born in Nis, Yugoslavia, on October 16, 1970. He received the B.E. degree in electrical engineering from the Faculty of Electronic Engineering, University of Nis in 1996.

His scientific interests include information theory and optical communications. 